\title{
Field enhancement properties of nanotubes in a field emission set-up
}

\author{
Ch. Adessi
}

NASA Ames Research center, Moffett Field CA, USA

\author{
M. Devel
}

Laboratoire de Physique Moléculaire, UMR CNRS 6624

Université de Franche-Comté, Besançon, France 


\section{Framework}

$\diamond$ Controversy in the mechanisms of emission

$\diamond$ Modeling of the polarization of CNT

- Resolution of the Poisson's equation

$\rightarrow$ Use of an atomic dipolar approximation

$\rightarrow$ The local field is computed with the LippmannSchwinger's equation

$\diamond \beta$ factor for SWNT:

- evolution with the length

- evolution with the diameter

- influence of the density

$\diamond \beta$ factor for MWNT

$\diamond$ Thanks and conclusions 


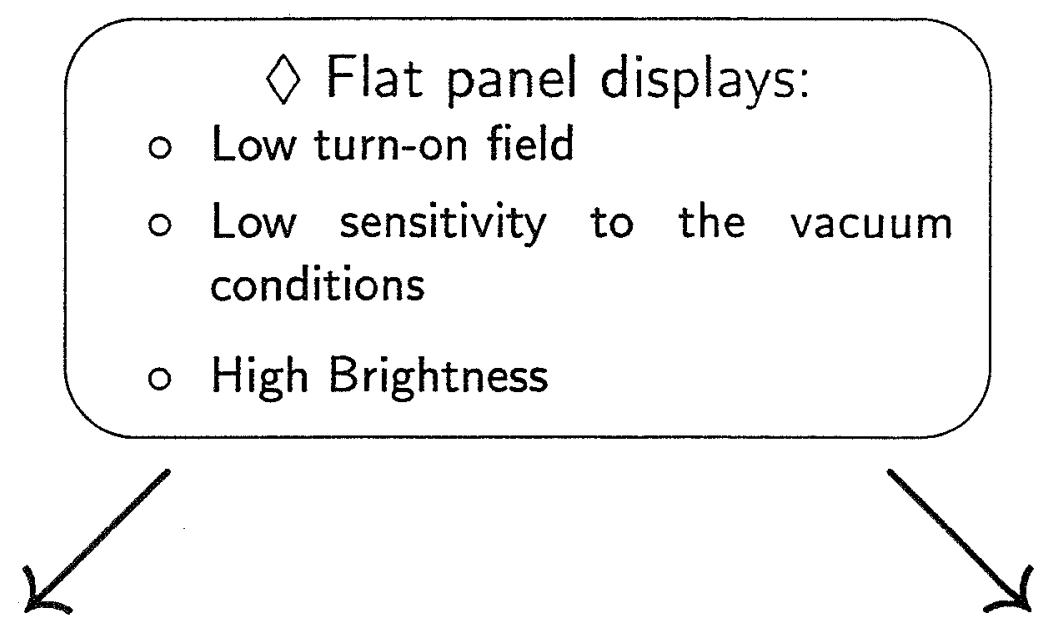

NEA materials:

Diamond type films:

- Emission from localized sites

- Emission mechanisns not well known

Ultra Thin SC film:

- Emission properties due to nanometer thickness

- Uniform emission

- Mechanism: Electronic injection $\rightarrow$ bending of the conduction band

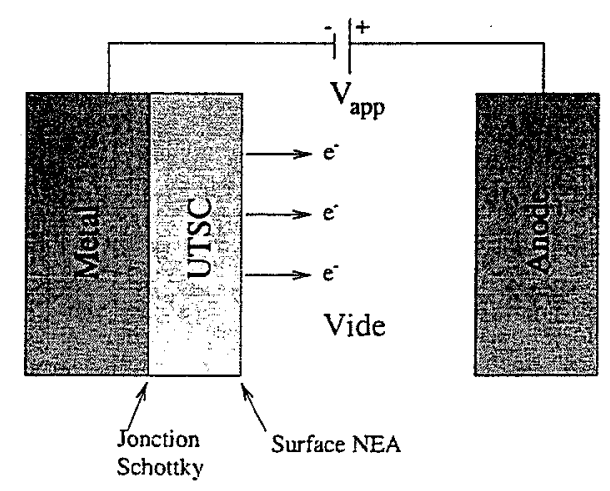

Vu Thien Binh, Adessi, PRL 85 (2000)

\section{Carbone nanotubes:}

- turn-on field $\leq 1 \mathrm{~V} / \mu \mathrm{m}$

- Prototype of display already achieved

Nanotubes forest:

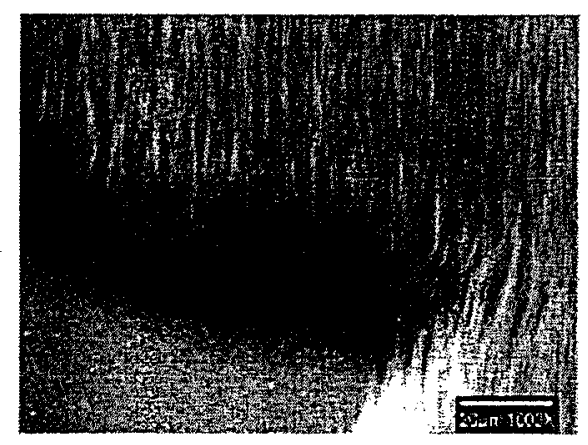

Z. F. Ren, Science 282 (1998)

Multi-wall Nanotubes:
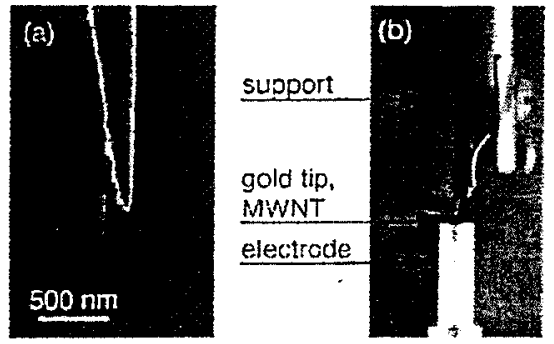

J.-M. Bonard, Appl. Phys. A 69 (1999) 


\section{Mechanisms of emission}

The mechanism leading to the electronic emission at low field is not well understood

$\Rightarrow$ Several phenomena are suspected to be involved

- Enhancement of the applied field:

$\rightarrow$ Polarization phenomenon

$\rightarrow$ Localized space charge

- Implication of localized states at the end of the nanotubes

- Uniform and atomic descriptions lead to contradictory results

- Is Fowler-Nordheim still valid?

\section{Energy Distributions}

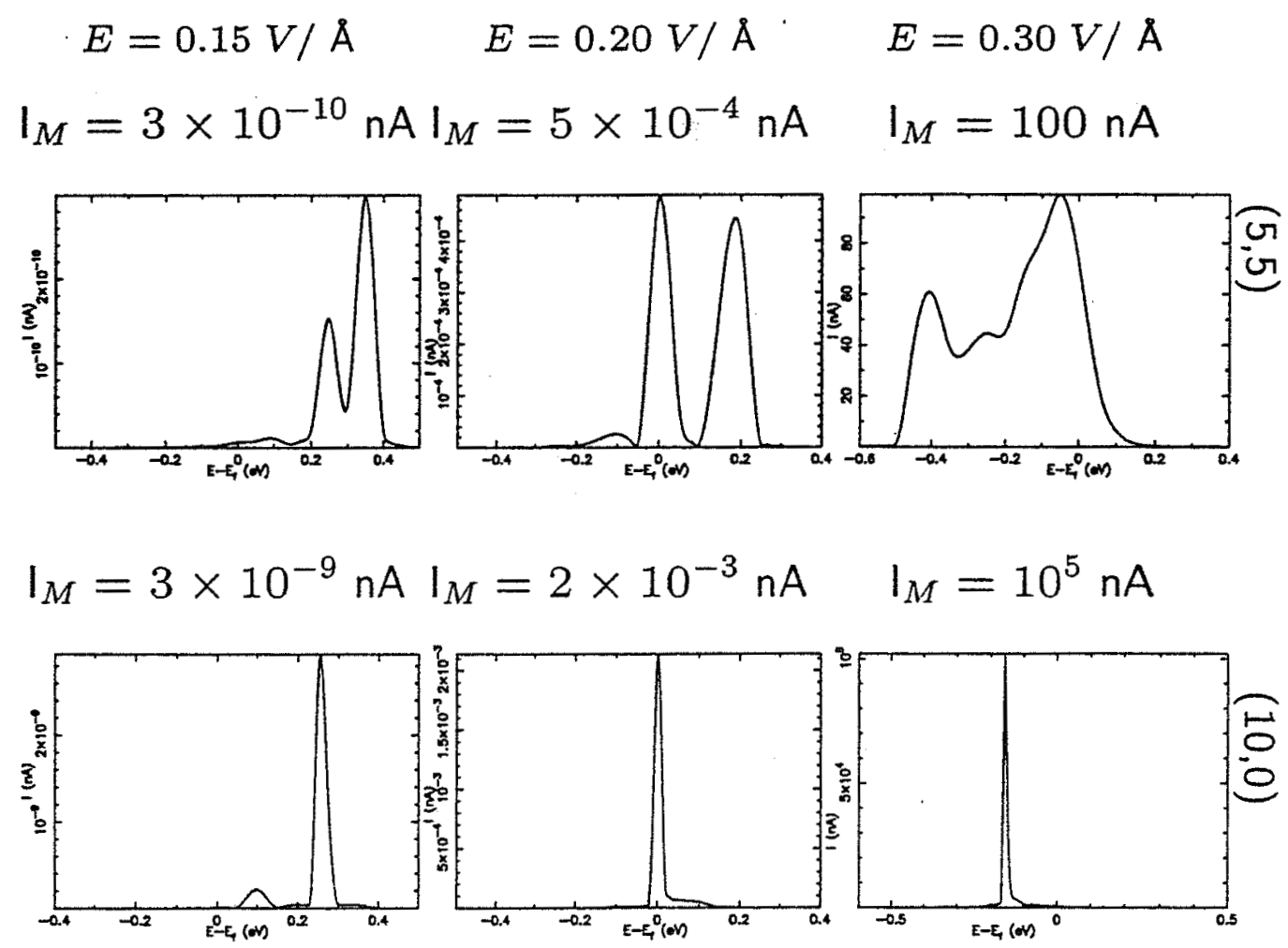




\section{Theoretical background}

$\diamond$ Aim: Compute electrostatic field near nanotube's end

$\diamond$ Model: Atomic dipoles and perfect metal

$\Rightarrow$ Self-consistent resolution of Maxwell-Gauss's law:

$$
\vec{\nabla}_{\vec{r}} \cdot \vec{E}(\vec{r})=-\frac{1}{\epsilon_{0}} \vec{\nabla} \cdot \vec{P}=\vec{\nabla}_{\vec{r}} \cdot\left[\sum_{j=1}^{N_{a t}}{\overleftrightarrow{\alpha_{j}}}_{\delta}\left(\vec{r}-\vec{r}_{j}\right) \vec{E}(\vec{r})\right]
$$

$\Rightarrow \vec{E}(\vec{r})$ solution of the LippmannSchwinger's equation:

$$
\begin{aligned}
\vec{E}(\vec{r})= & \vec{E}_{0}(\vec{r})+ \\
& \sum_{j=1}^{N_{a t}} \overleftrightarrow{S_{0}}\left(\vec{r}, \vec{r}_{j}\right) \cdot \overleftrightarrow{\alpha_{j}} \cdot \vec{E}\left(\vec{r}_{j}\right)
\end{aligned}
$$

$\diamond$ Local field $\rightarrow \beta=\frac{E_{l o c}}{E_{a p p}}=\frac{M a x\left(E_{z}\right)}{E_{0}}$

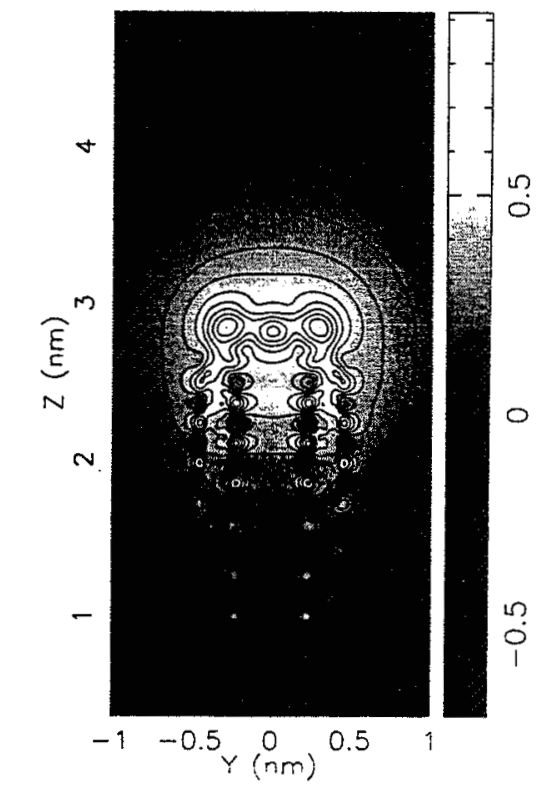

Polarization potential $(5,5)$ capped nanotube 


\section{Single wall nanotubes}
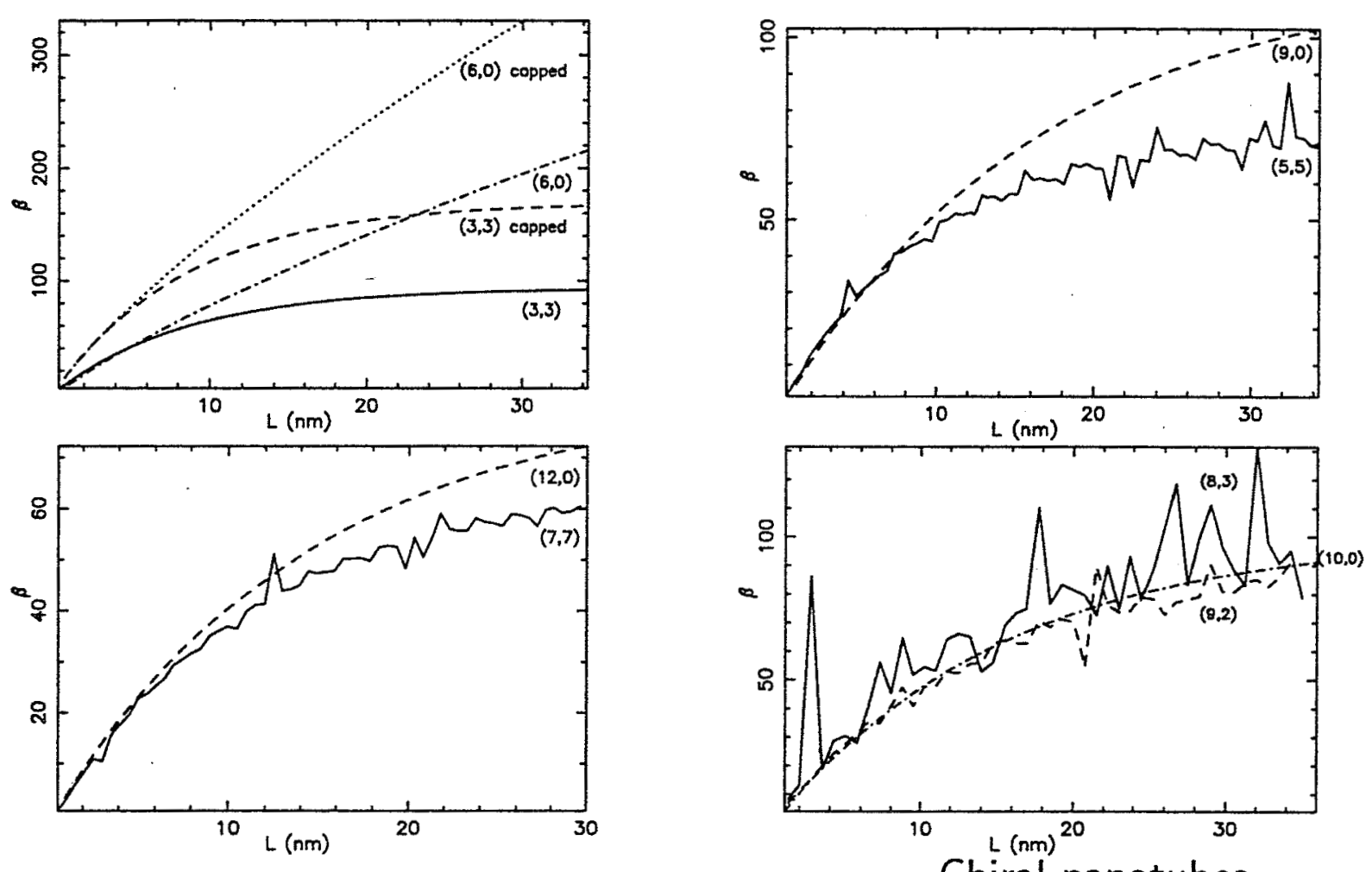

Chiral nanotubes

$$
\beta(L)=L \times\left[\begin{array}{c}
\text { general trend: } \\
\left.a_{0}+a_{1} \ln (L)+a_{2} \ln ^{2}(L)+\ldots\right]
\end{array}\right.
$$

$\diamond$ Saturation of the $\beta$ factor with the length

$\diamond$ Saturation sets up faster for $(n, n)$

$\diamond$ The caps improve the $\beta$ factor but do not modify the general trend

$\diamond$ No significant influence of the chiral angle

$\diamond$ Extrapolation for a length of $1 \mu \mathrm{m}:(6,0)$ capped $\rightarrow \beta \simeq 1100$ 


\section{Variation with the diameter}

$$
L=30 \mathrm{~nm} \quad L=1 \mu \mathrm{m} \text { (extrapolated) }
$$
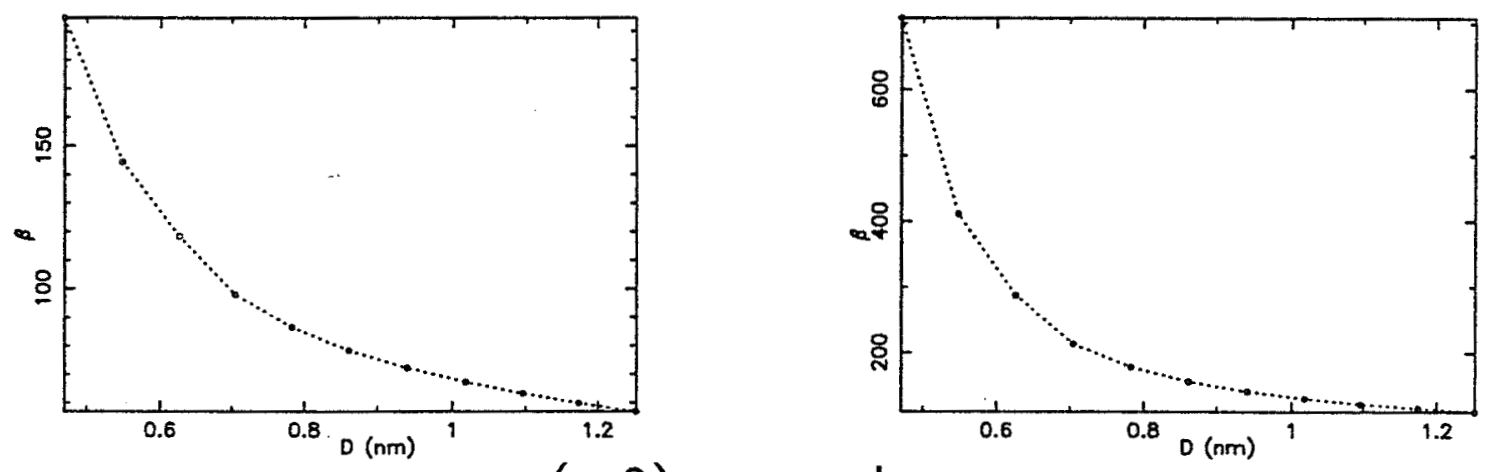

$(n, 0)$ nanotubes

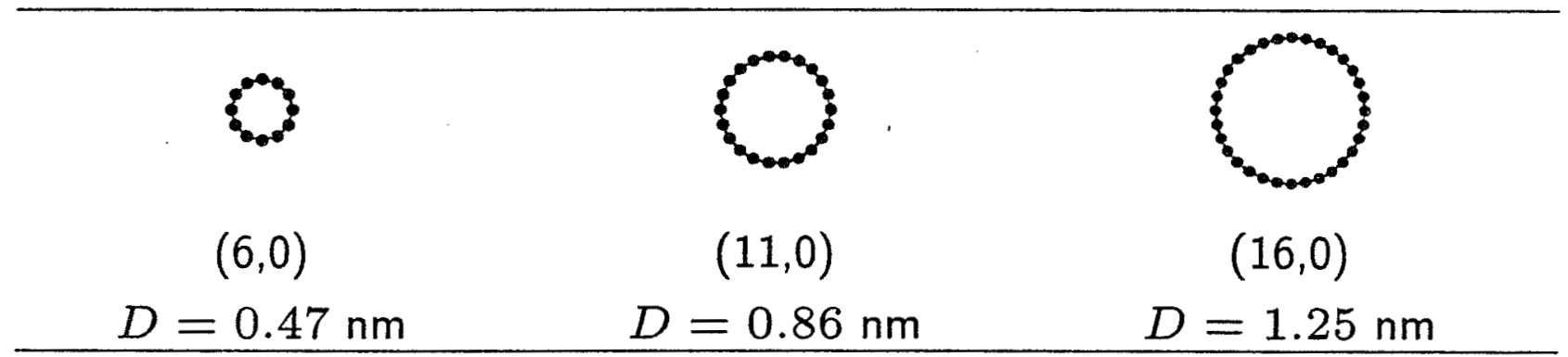

$\diamond$ Variation law:

$$
\beta(D)=a_{0}+\frac{a_{1}}{D}+\frac{a_{2}}{D^{2}}+\frac{a_{3}}{D^{3}}+\ldots
$$

$\diamond \beta \geq 1000$ can only be obtained with $D \leq 0.5 \mathrm{~nm}$

$\diamond$ For a $(9,0), \beta$ is only around 200

$\Rightarrow$ Phenomena other than polarization are probably involved in the emission 


\section{Influence of the density}
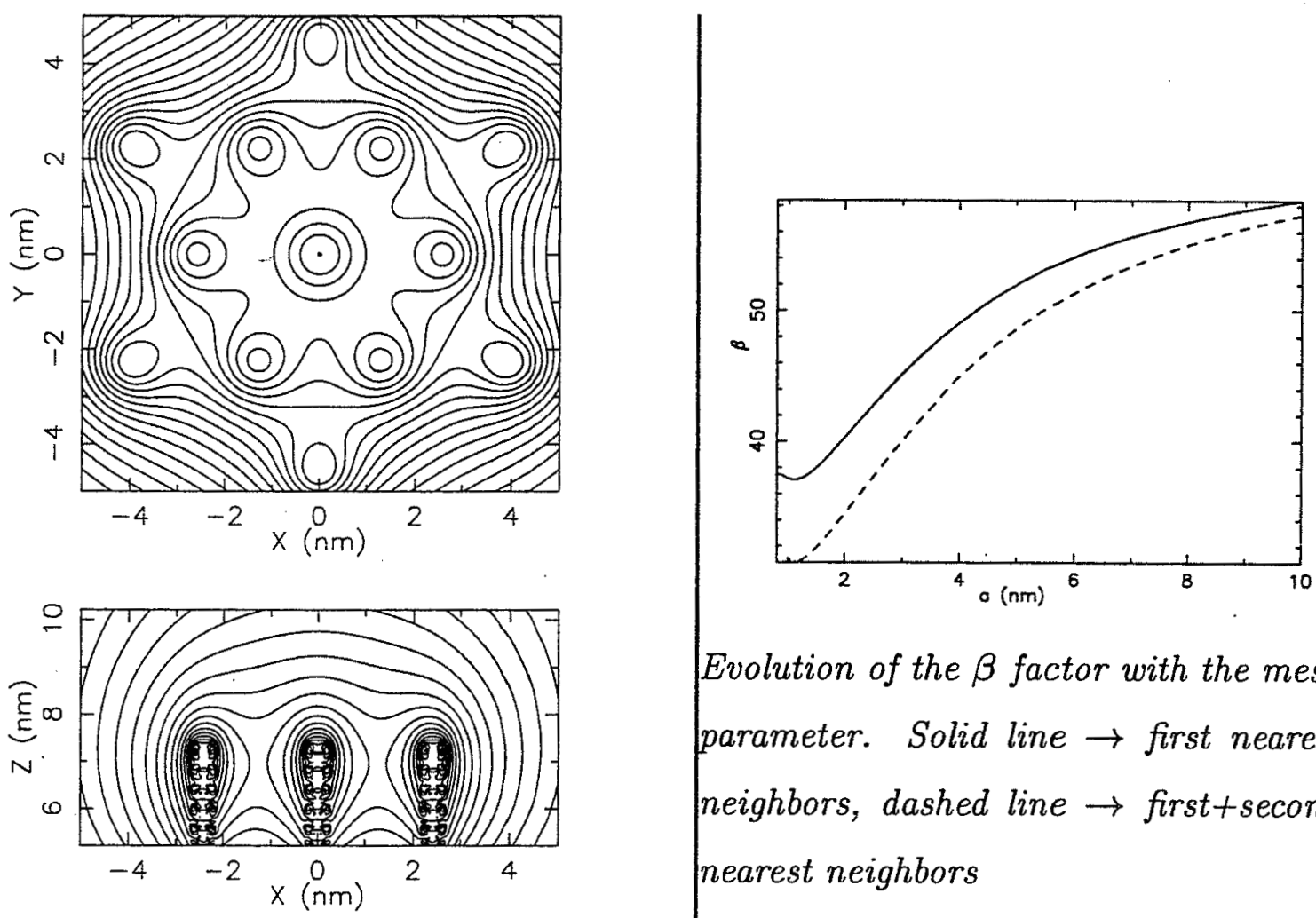

Evolution of the $\beta$ factor with the mesh parameter. Solid line $\rightarrow$ first nearest neighbors, dashed line $\rightarrow$ first+second nearest neighbors

Polarization potential in the $X Y$ plane (top) at $Z=8.5 \mathrm{~nm}$ and $X Z$ plane (bottom) at $Y=0$ for a rope of $13(6,0)$ nanotubes

$\diamond$ The $\beta$ factor decreases when the density is increased

$\Rightarrow$ To have a uniform emission $\rightarrow$ decrease the density

$\diamond$ The polarization is larger for the external nanotubes

$\diamond$ The $X Y$ components of the field are larger than the $Z$ component

$\Rightarrow$ Emission from the brim of the rope $\rightarrow$ large opening of the beam 


\section{Multi wall nanotubes}

$(3,3) @(13,5)$ and $(3,3) @(15,2)$

$(9,0) @(19,1)$ and $(9,0) @(15,7)$
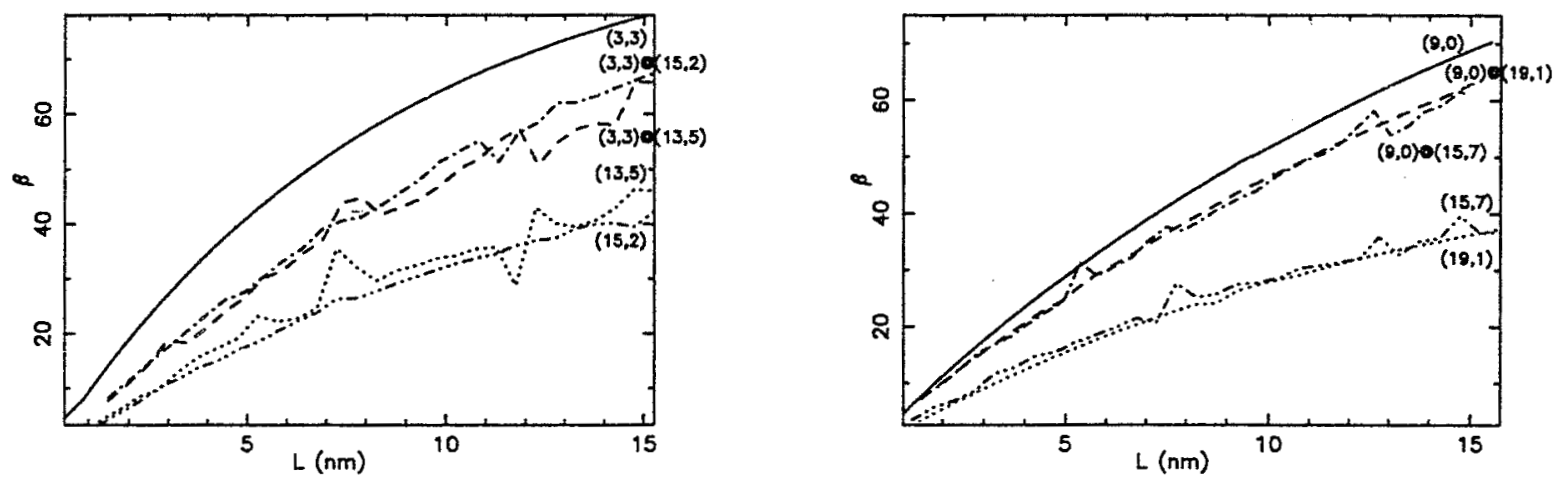

$(3,3)$

$(15,2)$

$(24,4)$

30

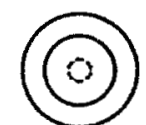

$(3,3) @(15,2) @(24,4)$
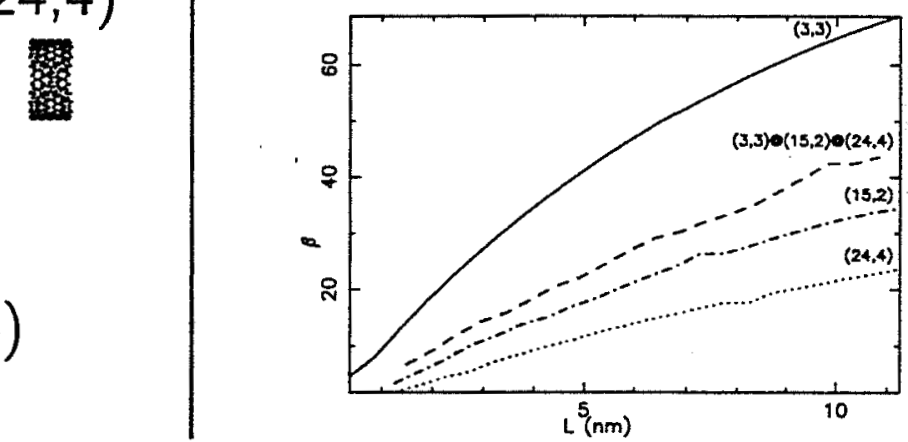

$\diamond$ No significant influence of chirality

$\diamond$ The maximum value of the $\beta$ factor is given by the inner shell

$\diamond$ The addition of shells tends to sweep out the instabilities of the $\beta$ factor

$\diamond$ The outer shells tend to reduce the enhancement property of the inner shell $\rightarrow$ Faraday cages 


\section{Conclusions}

$\Rightarrow$ No influence of the band structure on the polarization

$\Rightarrow$ Saturation of the polarization with the length of the nanotubes

$\Rightarrow$ The saturation sets up faster for $(n, n)$ nanotubes

$\Rightarrow(\mathrm{n}, 0)$ nanotubes are the best field amplifier

$\Rightarrow$ The largest $\beta$ factor observed for isolated SWNT is only of the order of $200 \rightarrow$ other phenomena are probably involved

$\Rightarrow$ In the case of a rope, the induced field is larger close to the brim

$\Rightarrow$ The field amplification of MWNT seems to be due to small inner tubes

The authors gratefully thank J.-M. Bonard (EPFL Lausanne) for numerous dicussions. 